\title{
Efficient Numerical Methods for Analysis of Square Ratio of $\kappa-\mu$ and $\eta-\mu$ Random Processes with Their Applications in Telecommunications
}

\author{
Gradimir V. Milovanović $\left(\mathbb{D},{ }^{1,2}\right.$ Suad Suljović, ${ }^{3}$ Stefan R. Panić $\left(\mathbb{D},{ }^{4}\right.$ \\ Ismet Kalčo, ${ }^{5}$ and Mihajlo Č. Stefanović $\mathbb{1}^{3}$ \\ ${ }^{1}$ Serbian Academy of Sciences and Arts, Kneza Mihaila 35, 11000 Beograd, Serbia \\ ${ }^{2}$ Faculty of Sciences and Mathematics, University of Niš, P.O. Box 224, 18000 Niš, Serbia \\ ${ }^{3}$ Department of Telecommunications, Faculty of Electrical Engineering, University of Niš, $18000 \mathrm{Niš}$, Serbia \\ ${ }^{4}$ Department of Informatics, Faculty of Natural Science and Mathematics, University of Priština, \\ 383200 Kosovska Mitrovica, Serbia \\ ${ }^{5}$ Faculty of Polytechnics, University of Zenica, Zenica, Bosnia and Herzegovina
}

Correspondence should be addressed to Stefan R. Panić; stefanpnc@yahoo.com

Received 12 October 2017; Accepted 30 January 2018; Published 11 March 2018

Academic Editor: Bo Shen

Copyright (C) 2018 Gradimir V. Milovanović et al. This is an open access article distributed under the Creative Commons Attribution License, which permits unrestricted use, distribution, and reproduction in any medium, provided the original work is properly cited.

We will provide statistical analysis of the square ratio of $\kappa-\mu$ and $\eta-\mu$ random processes and its application in the signal-tointerference ratio (SIR) based performance analysis of wireless transmission subjected to the influence of multipath fading, modelled by $\kappa-\mu$ fading model, and undesired occurrence of co-channel interference (CCI), distributed as $\eta-\mu$ random process. First contribution of the paper is deriving exact closed expressions for the probability density function (PDF) and cumulative distribution function (CDF) of square ratio of $\kappa-\mu$ and $\eta-\mu$ random processes. Further, a verification of accuracy of these $\mathrm{PDF}$ and CDF expressions was given by comparison with the corresponding approximations obtained by the high-precision quadrature formulas of Gaussian type with respect to the weight functions on $(0,+\infty)$. The computational procedure of such quadrature rules is provided by using the constructive theory of orthogonal polynomials and the Mathematica package OrthogonalPolynomials created by Cvetković and Milovanović (2004). Capitalizing on obtained expression, important wireless performance criteria, namely, outage probability (OP), have been obtained, as functions of transmission parameters. Also, possible performance improvement is observed through a glance at SC (selection combining) reception employment based on obtained expressions.

\section{Introduction}

Let $X_{1 i}$ and $X_{2 i}$ be mutually independent Gaussian random processes, with variances $V\left(X_{1 i}\right)=V\left(X_{2 i}\right)=\sigma^{2}$. Modelling mean values of Gaussians, with $p_{i}$ and $q_{i}$, and assuming $\mu$ initially to be an integer, the resulting random process $x$ is obtained as the following function of Gaussians:

$$
x=\sqrt{\sum_{i=1}^{\mu}\left(X_{1 i}+p_{i}\right)^{2}+\sum_{i=1}^{\mu}\left(X_{2 i}+q_{i}\right)^{2}}
$$

which follows $\kappa-\mu$ distribution, whose PDF is given in the following form (cf. [1]):

$$
\begin{aligned}
f_{X}(x)= & \frac{2 \mu(1+\kappa)^{(\mu+1) / 2} x^{\mu}}{\kappa^{(\mu-1) / 2} \mathrm{e}^{\mu \kappa} \Omega^{(\mu+1) / 2}} \exp \left(-\frac{\mu(1+\kappa) x^{2}}{\Omega}\right) \\
& \cdot I_{\mu-1}\left[2 \mu \sqrt{\frac{\kappa(1+\kappa) x^{2}}{\Omega}}\right],
\end{aligned}
$$


with

$$
\begin{aligned}
p^{2} & =\sum_{i=1}^{\mu} p_{i}^{2}, \\
q^{2} & =\sum_{i=1}^{\mu} q_{i}^{2}, \\
\kappa & =\frac{p^{2}+q^{2}}{2 \mu \sigma^{2}}, \\
\Omega & =2 \mu \sigma^{2}(1+\kappa),
\end{aligned}
$$

and $I_{0}(x)$ is the zero-the order modified Bessel function of the first kind [2, Eq. 8.445].

As a general probability distribution model, this model includes some classical distribution models as its particular cases, for example, Rician, and Nakagami- $m$ distribution models as special cases (as the One-Sided Gaussian and the Rayleigh distributions since they also represent special cases of Nakagami- $m$ ).

Let $Y_{1 i}$ and $Y_{2 i}, i=1, \ldots, \mu$, be Gaussian random processes, with $E\left(Y_{1 i}\right)=E\left(Y_{2 i}\right)=0$. Assuming $\mu$ initially to be an integer, resulting random process $y$ obtained as a function of Gaussians

$$
Y=\sqrt{\sum_{i=1}^{\mu}\left(Y_{1 i}^{2}+Y_{2 i}^{2}\right)}
$$

follows $\eta-\mu$ distribution, whose PDF is given in the following form (cf. [1]):

$$
\begin{aligned}
f_{Y}(y)= & \frac{4 \sqrt{\pi} \mu^{\mu+1 / 2} h^{\mu} y^{2 \mu}}{\Gamma(\mu) H^{\mu-1 / 2} \Omega^{\mu+1 / 2}} \exp \left(-\frac{2 \mu h y^{2}}{\Omega}\right) \\
& \cdot I_{\mu-1 / 2}\left[\frac{2 \mu H y^{2}}{\Omega}\right]
\end{aligned}
$$

with $\Omega$ defined as $\Omega=E\left(y^{2}\right)$, the ratio of arbitrary variances of independent Gaussians defined as $\eta=E\left(Y_{1 i}^{2}\right) / E\left(Y_{2 i}^{2}\right)$, and distribution parameters $H$ and $h$ defined as $H=$ $\left(\eta^{-1}-\eta\right) / 4, h=\left(2+\eta^{-1}+\eta\right) / 4, \eta \geq 0$. As a general probability distribution model, this model also includes some classical distribution models as its particular cases, for example, Nakagami- $q$ (Hoyt), one-sided Gaussian, Rayleigh, and Nakagami-m.

Multipath fading is physical phenomena that occurs as randomly delayed components of desired signal combine in constructive or destructive manner at the reception [1]. In the case when a line of sight (LOS) component between the transmitter and the receiver is present, the most general fading model which describes the short-term signal variation is kappa-mu $(\kappa-\mu)$ fading model $[3,4]$. Fading $\kappa-\mu$ model, defined as the function of parameter $\kappa$, related to the dominant/scattered components powers quotient, and parameter $\mu$, related to the number of propagating clusters, easily reduces to other fading models, by setting corresponding values for parameters $\kappa$ and $\mu$. When $\mu=1$, the $\kappa-\mu$ model transforms to the Rice model with arbitrary $K$ factor, $K=\kappa$ [4]. By assigning $\kappa=0$, the observed $\kappa-\mu$ model transforms to the Nakagami- $m$ model [4]. Co-channel interference (CCI) signal which is transmitted at the same frequency as the carrier signal is also exposed to multipath fading phenomenon [5]. Due to complicated propagation of interfering signal, CCI at the reception is usually modelled with random process that describes small scale signal variations in general nonline-of-sight condition. Recently proposed [6] eta-mu $(\eta-\mu)$ fading distribution meets those conditions. As mentioned in [7], $\eta-\mu$ model is presented in function of the parameter $\eta$, related to the in-phase/in-quadrature components scattered wave powers quotient of each cluster, and parameter $\mu$, related to the number of multipath clusters in the environment. As a general distribution, this model spans through other some well-known fading models as its particular cases, that is, Hoyt model and Nakagami- $m$ model. Namely, Nakagami- $m$ distribution can be obtained in an exact manner by assigning parameter values $\mu=m$ and $\eta \rightarrow 0$ (cf. [6]).

Observing interference-limited fading environment, where corruptive effects of thermal noise can be ignored, it is necessary to determine properties of signal-to-interference ratio (SIR) in order to carry out effective performance analysis of observed system. Indeed, SIR has been measured in base and mobile stations by using SIR estimators [8]. In order to determine behavior of instantaneous SIR random process, we must determine square ratio of $\kappa-\mu$ and $\eta-\mu$ random processes, $\lambda=X^{2} / Y^{2}$, and analyze its properties. Here, exact closed expressions for the probability density function (PDF) and cumulative distribution function (CDF) of square ratio of $\kappa-\mu$ and $\eta-\mu$ random processes are shown. Further, a verification of accuracy of these PDF and CDF expressions was given by comparing with the corresponding approximations obtained by the high-precision quadrature formulas of Gaussian type with respect to the weight functions on $(0,+\infty)$. The computational procedure of such quadrature rules is provided by using Gautschi's constructive theory of orthogonal polynomials (see $[9,10]$ ) and the Mathematica package OrthogonalPolynomials created by Cvetković and Milovanović (see [11, 12]). Based on obtained expressions, rapidly converging infinite-series expression for wireless communication systems performance measure, outage probability (OP) is also presented, and OP is considered for different values of transmission parameters. At the end, possible performance improvement will be considered through a glance at SC (selection combining) reception employment.

The paper is organized as follows. System model and closed-form of the PDF and CDF expressions are presented in Section 2. Section 3 is devoted to an alternative approach based on the constructive theory of orthogonal polynomials and the corresponding quadrature rules of Gaussian type. System performances and numerical results are presented in Sections 4 and 5, respectively. Finally, concluding comments are given in Section 6. 


\section{System Model and Closed-Form of the PDF and CDF Expressions}

The desired signal follows $\kappa-\mu$ distributed random process modelled as (cf. [3])

$$
\begin{aligned}
p_{R}(R)= & C_{R} R^{\mu_{d}} \exp \left(-\frac{\mu_{d}\left(1+\kappa_{d}\right) R^{2}}{\Omega_{d}}\right) \\
& \cdot I_{\mu_{d}-1}\left[2 \mu_{d} \sqrt{\frac{\kappa_{d}\left(1+\kappa_{d}\right) R^{2}}{\Omega_{d}}}\right],
\end{aligned}
$$

where

$$
C_{R}=\frac{2 \mu_{d}\left(1+\kappa_{d}\right)^{\left(\mu_{d}+1\right) / 2}}{\kappa_{d}^{\left(\mu_{d}-1\right) / 2} \mathrm{e}^{\mu_{d} \kappa_{d}} \Omega_{d}^{\left(\mu_{d}+1\right) / 2}},
$$

with $\Omega_{d}=E\left[R^{2}\right]$, denoting desired signal average power, while $I_{\nu}(x)$ being the $v$ th order modified Bessel function of the first kind [2, Eq. 8.445]. Due to physical separation between the co-channel interferer and target receiver, which is very large in practice, it is very unlikely that direct LOS condition (s) exist, for CCI signal. The most general fading model, which does not include LOS condition is $\eta-\mu$ fading model, is chosen here for modelling random envelope fluctuations of CCI signal with corresponding PDF [6]:

$$
\begin{aligned}
p_{r}(r)= & \frac{4 \sqrt{\pi} \mu_{c}{ }^{\mu_{c}+1 / 2} h_{c}{ }^{\mu_{c}} r^{2 \mu_{c}}}{\Gamma\left(\mu_{c}\right) H_{c}{ }^{\mu_{c}-1 / 2} \Omega_{c}{ }^{\mu_{c}+1 / 2}} \exp \left(-\frac{2 \mu_{c} h_{c} r^{2}}{\Omega_{c}}\right) \\
& \cdot I_{\mu_{c}-1 / 2}\left[\frac{2 \mu_{c} H_{c} r^{2}}{\Omega_{c}}\right],
\end{aligned}
$$

where $\Omega_{c}=E\left[R^{2}\right]$ stands for the average power of CCI, while $\Gamma(a)$ denotes Gamma function [2, Eq. 8.310.1]. Here $H_{c}$ and $h_{c}$ are interfering signal parameters, written in the function of parameter $\eta_{c}$ as follows [6]:

$$
\begin{aligned}
& H_{c}=\frac{\eta_{c}^{-1}-\eta_{c}}{4}, \\
& h_{c}=\frac{2+\eta_{c}^{-1}+\eta_{c}}{4} .
\end{aligned}
$$

Now, we will observe random envelope statistics in observed interference-limited system. Here the novelty and significance of this model must be pointed out again. Namely, for the first time scenario is observed when desired signal envelope variations are caused by $\kappa-\mu$ fading occurrence (most general fading model which includes LOS component existence), while CCI signal envelope variations are caused by $\eta-\mu$ fading occurrence (most general fading model that does not include LOS component). Previously, various other scenarios have been observed in $[1,13-15]$. In $[1,14]$ LOS component existence in CCI signal has been assumed, which is not so common case, because of large physical distance between the interferer and receiver. In [13] non-LOS model was observed for desired signal, while Gamma approximation of $\eta-\mu$ random variables sum has been observed. Finally, neither in general nor in comprehensive study, such as [15], has this model been considered. In the case when CCI is much stronger than Gaussian noise in same channel, PDF for the instantaneous SIR, $\lambda=R^{2} / r^{2}$, can be determined according to [16] in the form

$$
p_{\lambda}(t)=\frac{1}{2 \sqrt{t}} \int_{0}^{+\infty} p_{R}(r \sqrt{t}) p_{r}(r) r \mathrm{~d} r .
$$

After substituting (6) and (8) into (10), we can derive PDF of SIR in the following closed-form:

$$
\begin{aligned}
& p_{\lambda}(\lambda)=\frac{2 \sqrt{\pi} h_{c}^{\mu_{c}}}{\exp \left(\mu_{d} \kappa_{d}\right) \Gamma\left(\mu_{c}\right)} \\
& \cdot \sum_{p=0}^{\infty} \sum_{\ell=0}^{\infty} A_{p l} \mu_{d}^{2 p+\mu_{d}}\left(1+\kappa_{d}\right)^{p+\mu_{d}} \kappa_{d}^{p} \mu_{c}^{2 \ell+2 \mu_{c}} H_{c}^{2 \ell} \\
& \times \frac{(S / \lambda)^{2 \ell+2 \mu_{c}+1}}{S\left[\mu_{d}\left(1+\kappa_{d}\right)+2(S / \lambda) \mu_{c} h_{c}\right]^{p+2 \ell+2 \mu_{c}+\mu_{d}}}
\end{aligned}
$$

where

$$
A_{p l}=\frac{\Gamma\left(p+2 \ell+2 \mu_{c}+\mu_{d}\right)}{\Gamma\left(\mu_{d}+p\right) \Gamma\left(\mu_{c}+\ell+1 / 2\right) p ! \ell !}
$$

and $S=\Omega_{d} / \Omega_{c}$ stands for the average SIR.

Namely, with respect to well-known series representation of modified Bessel function (cf. [2, Eq. 8.445])

$$
I_{\nu}(z)=\sum_{k=0}^{+\infty} \frac{1}{k ! \Gamma(k+v+1)}\left(\frac{z}{2}\right)^{2 k+v},
$$

substituting (6) and (8) in (10) results in

$$
\begin{aligned}
& p_{\lambda}(\lambda)=\frac{2 \sqrt{\pi} h_{c}^{\mu_{c}}}{\exp \left(\mu_{d} \kappa_{d}\right) \Gamma\left(\mu_{c}\right)} \\
& \quad \sum_{p=0}^{\infty} \sum_{\ell=0}^{\infty} \frac{\mu_{d}^{2 p+\mu_{d}}\left(1+\kappa_{d}\right)^{p+\mu_{d}} \kappa_{d}^{p} \mu_{c}^{2 \ell+2 \mu_{c}} H_{c}^{2 \ell}}{\Gamma\left(\mu_{d}+p\right) \Gamma\left(\mu_{c}+\ell+1 / 2\right) p ! \ell !} \\
& \times \frac{[S / \lambda)^{2 \ell+2 \mu_{c}+1}}{S\left(\mu_{d}\left(1+\kappa_{d}\right)+2(S / \lambda) \mu_{c} h_{c}\right]^{p+2 \ell+2 \mu_{c}+\mu_{d}}} \\
& \quad \times \int_{0}^{\infty} t^{p+2 \ell+2 \mu_{c}+\mu_{d}-1} \exp (-t) d t .
\end{aligned}
$$

By using the well-known definition of the Gamma function [2, Eq. 8.310.1]:

$$
\Gamma(a)=\int_{0}^{\infty} t^{a-1} \exp (-t) \mathrm{d} t
$$

(10) can be reduced into (11). A similar procedure has been used in [7]. By taking finite numbers of terms $p=\ell=m$ in 
TABLE 1: Number of terms that should be added in expression (18) in order to reach accuracy at 5th significant digit.

\begin{tabular}{ccccccc}
\hline$\mu_{d}$ & $\mu_{c}$ & $\kappa_{d}$ & $\kappa_{c}$ & $S=-10 \mathrm{~dB}$ & $S=0 \mathrm{~dB}$ & 22 \\
2.5 & 1.5 & 2.0 & 1.5 & 22 & 21 & 21 \\
2.5 & 2.5 & 2.0 & 1.5 & 21 & 20 & 20 \\
2.5 & 2.5 & 1.5 & 1.5 & 20 & 19 & 19 \\
2.5 & 2.5 & 1.5 & 0.5 & 19 & 19 \\
\hline
\end{tabular}

the last expression for $p_{\lambda}(\lambda)$ we obtain its approximation in a closed-form:

$$
\begin{aligned}
& p_{\lambda}(\lambda)_{m}=\frac{2 \sqrt{\pi} h_{c}^{\mu_{c}}}{\exp \left(\mu_{d} \kappa_{d}\right) \Gamma\left(\mu_{c}\right)} \sum_{p=0}^{m} \sum_{\ell=0}^{m} A_{p l} \\
& \times \frac{\mu_{d}^{2 p+\mu_{d}}\left(1+\kappa_{d}\right)^{p+\mu_{d}} \kappa_{d}^{p} \mu_{c}^{2 \ell+2 \mu_{c}} H_{c}^{2 \ell}(S / \lambda)^{2 \ell+2 \mu_{c}+1}}{S\left[\mu_{d}\left(1+\kappa_{d}\right)+2(S / \lambda) \mu_{c} h_{c}\right]^{p+2 \ell+2 \mu_{c}+\mu_{d}}} .
\end{aligned}
$$

The closed-form expression (16) converges rapidly, since only $m$ (10-15) terms are needed to be summed in each sum in order to reach an accuracy of five significant decimal digits, for observed set of system parameters. Otherwise, in a case of slowly convergent series, we need some methods for accelerating their convergence (cf. [17-19]).

Following [16], the cumulative distribution function (CDF) can be obtained as

$$
F_{\lambda}(t)=\int_{0}^{t} p_{\lambda}(x) \mathrm{d} x
$$

After substituting (11) into (17), by applying the same mathematical transformations given in Appendix of [7], we can obtain the following closed-form expression of rapid convergence:

$$
F_{\lambda}(t)=\frac{\sqrt{\pi}}{\mathrm{e}^{\mu_{d} k_{d}} \Gamma\left(\mu_{c}\right)} \sum_{p=0}^{\infty} \sum_{l=0}^{\infty} \frac{\mu_{d}{ }^{p} \kappa_{d}^{p} H_{c}^{2 \ell}}{2^{2 \ell+2 \mu_{c}-1} h_{c}^{2 \ell+\mu_{c}}} Q_{p, \ell},
$$

where

$$
\begin{aligned}
Q_{p, \ell} & =\frac{\Gamma\left(p+2 \ell+2 \mu_{c}+\mu_{d}\right) B_{z}\left(p+\mu_{d}, 2 \ell+2 \mu_{c}\right)}{\Gamma\left(\mu_{d}+p\right) \Gamma\left(\mu_{d}+\ell+1 / 2\right) p ! \ell !}, \\
z & =\frac{\lambda \mu_{d}\left(1+\kappa_{d}\right)}{\lambda \mu_{d}\left(1+\kappa_{d}\right)+2 S \mu_{c} h_{c}},
\end{aligned}
$$

and $B_{z}(a, b)$ is the incomplete Beta function [2, Eq. 8.38] (for some extended special functions see [20]). Detailed convergence analysis of this expression is provided in Table 1.

\section{Method of Quadrature Sums}

In this section we give an alternative approach for getting very high-precision approximation of the probability density function (PDF), as well as one of the cumulative distribution function (CDF). Our goal is to obtain fast convergence of quadrature sums, $Q_{n}(f ; w)=\sum_{k=1}^{n} A_{k}^{(n)} f\left(x_{k}^{(n)}\right)$, for integrals on half-infinite ranges or finite intervals for functions of the form $F(x)=w(x) f(x)$, where $w(x)(\geq 0)$ is the socalled weight function and $f(x)$ is a smooth factor if it is possible. We use here a computational procedure for constructing quadratures of Gaussian type with respect to the weight functions $w(x)$ on $(0,+\infty)$, as well as ones on finite intervals, supposing the polynomial behavior of the function $f(x)$ (for nonpolynomial cases see [21, 22], and for some nonstandard Gaussian quadratures see [23-25]). Our computational procedure provides the ability to verify the accuracy of expressions obtained in the previous section. As we will see, these quadrature processes give much better accuracy of the considered integrals.

As already mentioned PDF of this random process could be efficiently expressed by using Gaussian quadrature formulas, namely, starting from (10), which can be rewritten in the following form:

$$
\begin{aligned}
& p_{\lambda}(\lambda) \\
& \quad=A \int_{0}^{+\infty} x^{\mu_{c}+\mu_{d} / 2} \mathrm{e}^{-x} I_{\mu_{c}-1 / 2}\left(\alpha_{c} x\right) I_{\mu_{d}-1}\left(\alpha_{d} \sqrt{x}\right) \mathrm{d} x,
\end{aligned}
$$

with parameters $\alpha_{c}, \alpha_{d}$, and $A$ depending on (being functions of) normalized $\operatorname{SIR}(z=S / \lambda)$ expressed as

$$
\begin{aligned}
& \alpha_{c}(z)=\frac{2 \mu_{c} H_{c} z}{\mu_{d}\left(1+\kappa_{d}\right)+2 \mu_{c} h_{c} z}, \\
& \alpha_{d}(z)=2 \mu_{d} \sqrt{\frac{\kappa_{d}\left(1+\kappa_{d}\right)}{\mu_{d}\left(1+\kappa_{d}\right)+2 \mu_{c} h_{c} z}}, \\
& A(z) \\
& =\frac{2 \sqrt{\pi} \mu_{c}^{\mu_{c}+1 / 2} h_{c}^{\mu_{c}} \mu_{d}\left(1+\kappa_{d}\right)^{\left(\mu_{d}+1\right) / 2} z^{\mu_{c}+3 / 2}}{H_{c}^{\mu_{c}-1 / 2} \Gamma\left(\mu_{c}\right) \kappa_{d}^{\left(\mu_{d}-1\right) / 2} \mathrm{e}^{\kappa_{d} \mu_{d}}\left[\mu_{d}\left(1+\kappa_{d}\right)+2 \mu_{c} h_{c} z\right]^{\mu_{d} / 2+\mu_{c}+1} S} .
\end{aligned}
$$

For calculating the weighted integral (20) we use the $n$ point generalized Gauss-Laguerre quadrature formula (cf. [26, pp. 324-328])

$$
\int_{0}^{+\infty} x^{s} \mathrm{e}^{-x} f(x) \mathrm{d} x=\sum_{k=1}^{n} A_{k}^{(n)} f\left(x_{k}^{(n)}\right)+R_{n}(f),
$$

with respect to the generalized Laguerre weight function $w(x)=w^{(s)}(x)=x^{s} \mathrm{e}^{-x}$ on $(0,+\infty)$, with a special selection of the parameter $s$. The quadrature parameters, the nodes $x_{k}^{(n)} \equiv x_{k}$, and the weights (Christoffel numbers) $A_{k}^{(n)} \equiv A_{k}$ can easily be constructed by the wellknown Golub-Welsch procedure [27] implemented in the MATHEMATICA package OrthogonalPolynomials (see [11, 12]). This package is freely downloadable from the website http://www.mi.sanu.ac.rs/ gvm/. 
In fact, the nodes $x_{k}$ are eigenvalues of the following symmetric tridiagonal Jacobi matrix (cf. [26, pp. 325-328]):

$$
J_{n}=\left[\begin{array}{ccccc}
\alpha_{0} & \sqrt{\beta_{1}} & & & \mathbf{0} \\
\sqrt{\beta_{1}} & \alpha_{1} & \sqrt{\beta_{2}} & & \\
& \sqrt{\beta_{2}} & \alpha_{2} & \ddots & \\
& & \ddots & \ddots & \sqrt{\beta_{n-1}} \\
\mathbf{O} & & & \sqrt{\beta_{n-1}} & \alpha_{n-1}
\end{array}\right],
$$

and the weight coefficients $A_{k}$ are given by $A_{k}=\beta_{0} v_{k, 1}^{2}, k=$ $1, \ldots, n$, where $v_{k, 1}$ is the first component of the eigenvector $\mathbf{v}_{k}=\left[\begin{array}{lll}v_{k, 1} & \cdots & v_{k, n}\end{array}\right]^{T}$ corresponding to the eigenvalue $x_{k}$ and normalized such that $\mathbf{v}_{k}^{T} \mathbf{v}_{k}=1$.

Remark 1. In some special cases for calculating the weight coefficients $A_{k}$ it is better to use the complete eigenvectors, instead of their first components. An analysis of such cases is given in [28] (see also [29, p. 339] for an application).

Elements in the Jacobi matrix (23) are recursion coefficients in the three-term recurrence relation

$$
\begin{aligned}
\pi_{k+1}(t) & =\left(t-\alpha_{k}\right) \pi_{k}(t)-\beta_{k} \pi_{k-1}(t) \quad(k=0,1, \ldots), \\
\pi_{0}(t) & =1, \\
\pi_{-1}(t) & =0,
\end{aligned}
$$

for polynomials orthogonal with respect to the generalized Laguerre weight function $w(x)=w^{(s)}(x)$ on $(0,+\infty)$ :

$$
\begin{aligned}
& \alpha_{k}=2 k+s+1 \quad(k=0,1, \ldots), \\
& \beta_{k}=k(k+s) \quad(k=1,2, \ldots) .
\end{aligned}
$$

The coefficient $\beta_{0}$ can be arbitrary, but it is convenient to put

$$
\beta_{0}=\int_{0}^{+\infty} w^{(s)}(x) \mathrm{d} x=\Gamma(s+1),
$$

in order to hold certain compact formulas, including the previously mentioned ones for Christoffel numbers $A_{k}, k=$ $1, \ldots, n$.

Remark 2. The recursion coefficients in the three-term recurrence relation (24) are known in explicit form only for some narrow classes of orthogonal polynomials, among which are the most famous classical orthogonal polynomials (Jacobi, the generalized Laguerre, and Hermite polynomials) (cf. [26, pp. 121-146], [30], [31, pp. 19-24]). In the case of the so-called strongly nonclassical polynomials, the recursion coefficients must be constructed numerically using moment information on a given weight function (cf. [32-34]). For details on Gautschi's constructive theory of orthogonal polynomials see $[9,10,35]$, [26, pp. 160-166].

Knowing the first $N$ recursion coefficients in (24), we can obtain the nodes $x_{k}$ and the weights $A_{k}$ in (22) for any number of nodes $n \leq N$.
It is known that if the integrand in the weighted integral (20) is smooth, then we can expect Gaussian quadrature applied to (20) to converge rapidly. According to the series representation (13), we see that $x \mapsto x^{-v} I_{\nu}(x)$ is an entire function, and, therefore, the function

$$
\begin{aligned}
x & \longmapsto g(x ; z) \\
& =A(z) \frac{I_{\mu_{c}-1 / 2}\left(\alpha_{c}(z) x\right)}{x^{\mu_{c}-1 / 2}} \cdot \frac{I_{\mu_{d}-1}\left(\alpha_{d}(z) \sqrt{x}\right)}{x^{\left(\mu_{d}-1\right) / 2}}
\end{aligned}
$$

is also entire. This means that, for calculating (20), this integral should be written in the following form:

$$
p_{\lambda}(\lambda)=\int_{0}^{+\infty} x^{2 \mu_{c}+\mu_{d}-1} \mathrm{e}^{-x} g(x ; z) \mathrm{d} x
$$

where $z=S / \lambda$ and $g(x ; z)$ is defined by (27), and then quadrature formulas are applied with respect to the generalized Gauss-Laguerre weight with the parameter $s=2 \mu_{c}+\mu_{d}-$ 1. by

Then the sequence of quadrature sums $\left\{p_{\lambda}^{(n)}(\lambda)\right\}_{n}$, defined

$$
p_{\lambda}^{(n)}(\lambda)=\sum_{k=1}^{n} A_{k}^{(n)} g\left(x_{k}^{(n)} ; z\right),
$$

shows a rapid convergence.

For example, for some typical parameters

$$
\begin{aligned}
\Omega_{d} & =1, \\
\mu_{d} & =2, \\
\kappa_{d} & =1, \\
\eta_{c} & =\frac{1}{2}, \\
\rho_{c} & =1, \\
\mu_{c} & =2.01, \\
H_{c} & =\frac{\eta_{c}^{-1}-\eta_{c}}{4}, \\
h_{c} & =\frac{2+\eta_{c}^{-1}+\eta_{c}}{4}, \\
S & =\frac{\Omega_{d}}{\Omega_{c}},
\end{aligned}
$$

and $\lambda \in(0,3)$, we present the graphic of $\lambda \mapsto p_{\lambda}^{(20)}(\lambda)$ in Figure 1 as an approximation of the function $\lambda \mapsto p_{\lambda}(\lambda)$.

Relative errors

$$
\operatorname{err}_{n}(\lambda)=\left|\frac{p_{\lambda}^{(n)}(\lambda)-p_{\lambda}(\lambda)}{p_{\lambda}(\lambda)}\right|
$$

are presented in log-scale in Figure 2, for five different numbers of nodes in the quadrature sums $(n=4,6,8$, 10 , and 12). As the exact PDF we take the approximation 


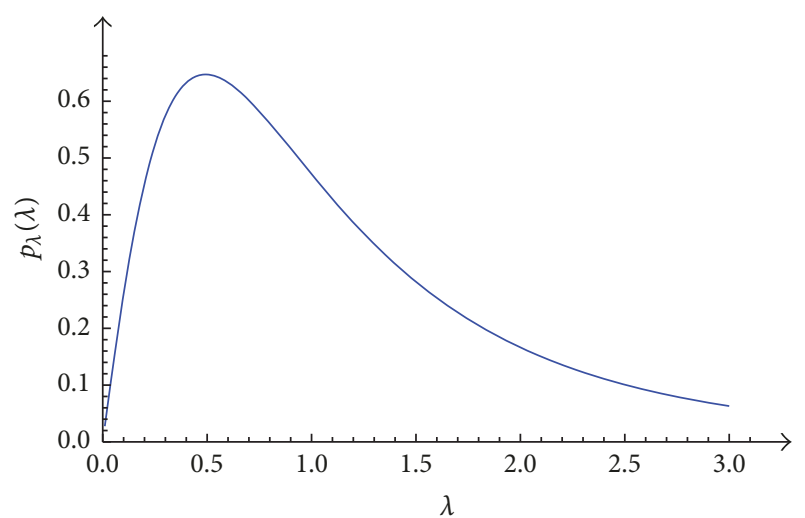

FIGURE 1: Approximation of PDF, $\lambda \mapsto p_{\lambda}(\lambda) \approx p_{\lambda}^{(n)}(\lambda)$, using $n=6$ nodes in (29).

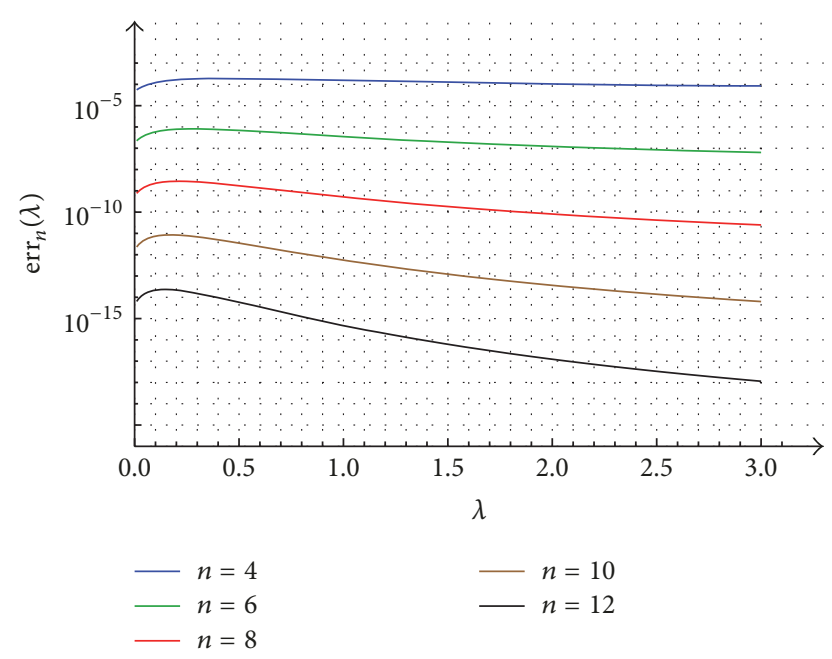

FIgURE 2: Relative errors in $p_{\lambda}^{(n)}(\lambda)$ obtained by the quadrature formula with $n=4(2) 12$ nodes.

$p_{\lambda}(\lambda) \approx p_{\lambda}^{(20)}(\lambda)$. For example, we can see that the quadrature sum with only six points gives an approximation of PDF for each $\lambda>0$, with the relative error less than $10^{-6}$; that is, $p_{\lambda}^{(6)}(\lambda)$ gives the result with at least six decimal digits, but the 10 -point quadrature sum approximates the PDF with at least eleven digits.

According to (17) the cumulative distribution function (CDF) can be transformed as

$$
F_{\lambda}(t)=t \int_{0}^{1} p_{\lambda}(t \tau) \mathrm{d} \tau
$$

For numerically calculating this integral we use the GaussLegendre quadrature rule (transformed to the interval $[0,1]$ ),

$$
\int_{0}^{1} f(\tau) \mathrm{d} \tau=\sum_{j=1}^{N} B_{j}^{(N)} f\left(\tau_{j}^{(N)}\right)+R_{N}(f),
$$

whose parameters, the nodes and weights, $\tau_{j}^{(N)}$ and $B_{j}^{(N)}, j=$ $1, \ldots, N$, can be obtained from the eigenvalue problem for the

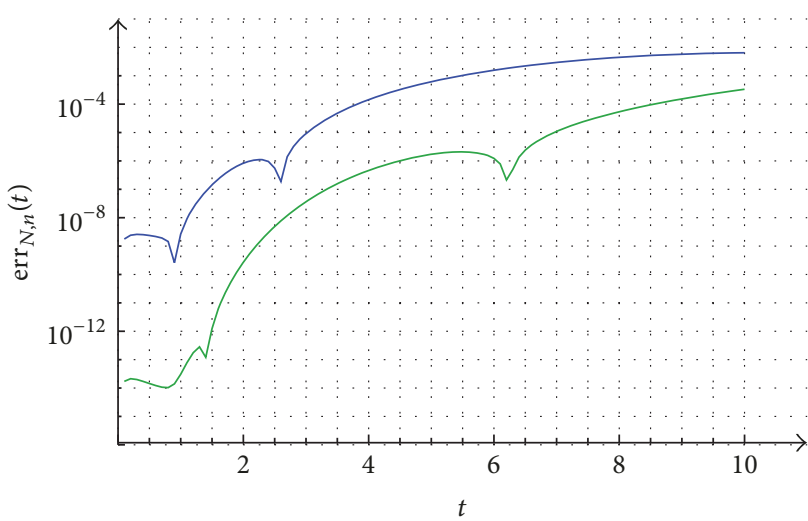

$\begin{aligned} N & =n=8 \\ N & =n=12\end{aligned}$

FIGURE 3: Relative errors in the quadrature sums $F_{\lambda}^{(N, n)}(t)$ for $N=$ $n=8$ and 12 nodes.

corresponding Jacobi matrix of the form (23), with recursion coefficients

$$
\begin{aligned}
& \alpha_{j}=\frac{1}{2} \quad(j=0,1, \ldots), \\
& \beta_{0}=1, \\
& \beta_{j}=\frac{j^{2}}{4\left(4 j^{2}-1\right)} \quad(j=1,2, \ldots) .
\end{aligned}
$$

In this symmetric case (with respect to $\tau=1 / 2$ ), it is clear that $\tau_{N+1-j}^{(N)}=1-\tau_{j}^{(N)}$ and $B_{N+1-j}^{(N)}=B_{j}^{(N)}, j=1,2, \ldots, N$. For a sufficiently smooth function $f \in C^{2 N}[0,1]$, the remaining term in (33) has the form [26, p. 333 \& p. 97]

$$
R_{N}(f)=\frac{\beta_{0} \beta_{1} \cdots \beta_{2 N}}{(2 N) !} f^{(2 N)}(\zeta) \quad(0<\zeta<1)
$$

As before, for approximating $p_{\lambda}(x)$, we use (29), so that we have

$$
F_{\lambda}^{(N, n)}(t)=t \sum_{j=1}^{N} B_{j}^{(N)} p_{\lambda}^{(n)}\left(t \tau_{j}^{(N)}\right)
$$

that is,

$$
F_{\lambda}^{(N, n)}(t)=t \sum_{j=1}^{N} B_{j}^{(N)} \sum_{k=1}^{n} A_{k}^{(n)} g\left(x_{k}^{(n)} ; \frac{S}{t \tau_{j}^{(N)}}\right) ;
$$

where $g(x ; z)$ is defined by (27).

The corresponding relative errors

$$
\operatorname{err}_{N, n}(t)=\left|\frac{F_{\lambda}^{(N, n)}(t)-F_{\lambda}(t)}{F_{\lambda}(t)}\right|
$$

are presented in log-scale in Figure 3 , for $N=n=8$ and 12 in the quadrature sums (36), that is, (37). As the exact CDF we used the approximation $F_{\lambda}(t) \approx F_{\lambda}^{(20,20)}(t)$. 


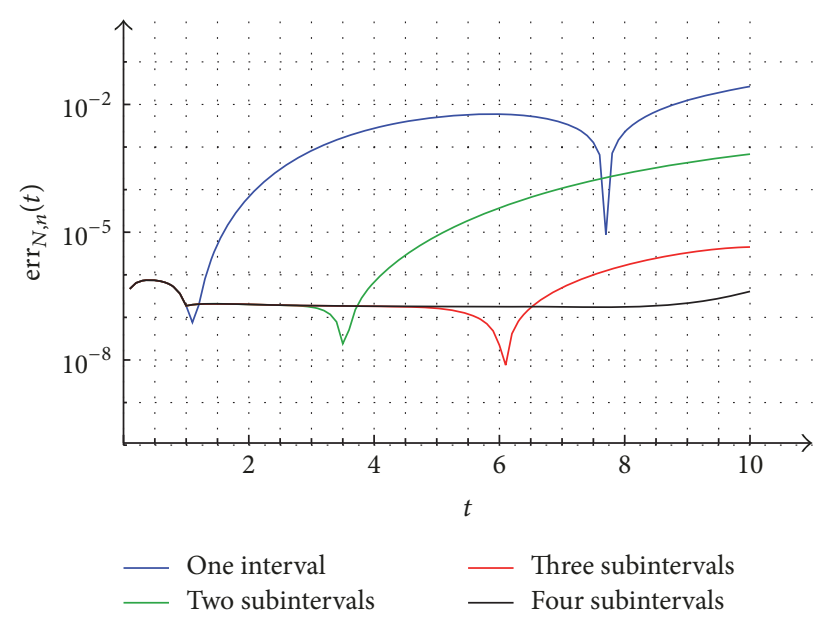

FIgURE 4: Relative errors in the quadrature sums $\widehat{F}_{\lambda}^{(N, n)}(t)$ for $0 \leq$ $t \leq 10$, taking $\nu+1$ subintervals $(\nu=0,1,2,3)$ and $N=n=6$.

As we can see, in the case when $t$ is large, this formula needs a bigger $N$, and therefore the following approach is much more convenient. Namely, we use a decomposition of the interval $[0, t]$ into several subintervals for larger values of $t$, for example,

$$
[0, t]=[0,1] \cup[1,2] \cup \cdots \cup[\nu-1, v] \cup[\nu, t]
$$

$$
(\nu \leq t),
$$

and then apply the Gauss-Legendre rule (33) to each of the subintervals. Thus,

$$
\begin{aligned}
& F_{\lambda}(t)=\sum_{i=1}^{v} \int_{i-1}^{i} p_{\lambda}(x) \mathrm{d} x+\int_{\nu}^{t} p_{\lambda}(x) \mathrm{d} x \\
& =\sum_{i=1}^{v} \int_{0}^{1} p_{\lambda}(\xi+i-1) \mathrm{d} \xi+(t-v) \int_{0}^{1} p_{\lambda}(\nu+(t \\
& -v) \xi) \mathrm{d} \xi=\int_{0}^{1}\left[\sum_{i=1}^{v} p_{\lambda}(\xi+i-1)\right. \\
& \left.+(t-v) p_{\lambda}(\nu+(t-v) \xi)\right] \mathrm{d} \xi
\end{aligned}
$$

where the sums on the right-hand side are empty for $t<$ $1(\nu=0)$. The corresponding quadrature approximation, analogous to (36), becomes

$$
\begin{gathered}
\widehat{F}_{\lambda}^{(N, n)}(t)=\sum_{j=1}^{N} B_{j}^{(N)}\left[\sum_{i=1}^{\nu} p_{\lambda}^{(n)}\left(\tau_{j}^{(N)}+i-1\right)\right. \\
\left.+(t-v) p_{\lambda}^{(n)}\left(\nu+(t-v) \tau_{j}^{(N)}\right)\right] .
\end{gathered}
$$

Taking $v+1$ subintervals $(v=0,1,2,3)$, the relative errors in the quadrature sums $\widehat{F}_{\lambda}^{(N, n)}(t)$ for $N=n=6$ are presented in Figure 4 for $0 \leq t \leq 10$. We see that the last case, with

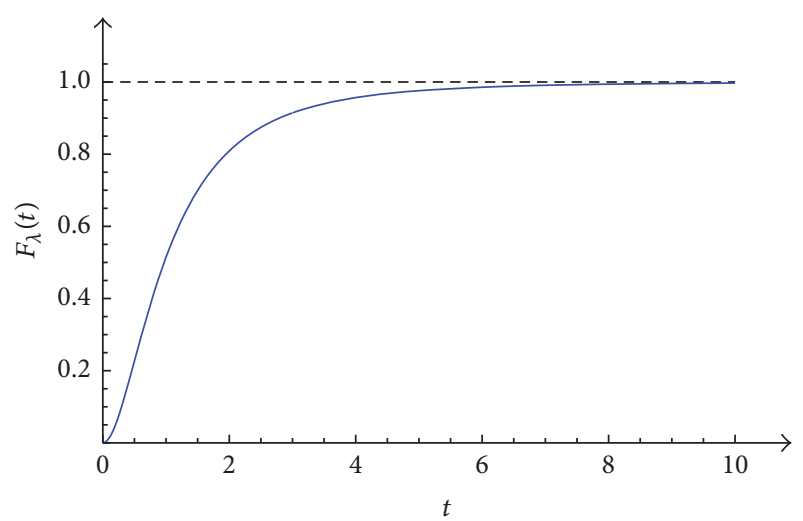

Figure 5: Approximation of CDF, $t \mapsto F_{\lambda}(t) \approx \widehat{F}_{\lambda}^{(N, n)}(t)$, for $N=$ $n=6$ with four subintervals $(\nu=3)$.

four subintervals $[0,1],[1,2],[2,3]$, and $[3, t](t \geq 3)$, gives the relative errors less than $10^{-6}$ for each $t \in[0,10]$. The corresponding cumulative distribution function is presented in Figure 5.

\section{System Performances}

One of standard performance criteria, used for managing CCI level in faded channel, and for meeting grade-of-service and quality-of-service requirements, is OP [36]. When considering cellular systems, OP is defined as the probability that received SIR falls below a given outage threshold $q_{\text {th }}$ also known as a protection ratio

$$
P_{\text {out }}=P_{R}\left(\sigma<q_{\mathrm{th}}\right)=\int_{0}^{q_{\mathrm{th}}} p_{\sigma}(t) \mathrm{d} t=F_{\sigma}\left(q_{\mathrm{th}}\right) .
$$

Diversity combining indicates a concept of upgrading reception system performances by combining replicas of information carrying signal [37]. By using separated antennas on reception diversity terminal, independent copies of desired signal are accomplished, without increase in signal bandwidth or transmitted signal power. Simplest space diversity combining method is selection combining (SC). In communication environments where the level of the CCI is significantly higher than the level of channel AWGN, SC outputs the antenna with the highest SIR. When reception system has uncorrelated branches (sufficient spacing between antennas), the PDF of the SIR at the output of the receiver is given by [38]:

$$
p_{\lambda}(t)=\sum_{j=1}^{N} p_{\lambda_{j}}(t) \prod_{i=1, i \neq j}^{N} F_{\lambda_{k}}(t),
$$

where $N$ denotes for the diversity reception order.

\section{Numerical Results}

At Figure $6 \mathrm{OP}$ in the function of normalized SIR in $\mathrm{dB}$ (decibels, $10 \log (S / \lambda)$ ) is presented. It is visible how smaller values of OP are obtained when parameter $\kappa_{d}$ arises, since dominant component of desired signal then straightens. Also better performance (smaller values of $\mathrm{OP}$ ) is obtained when 


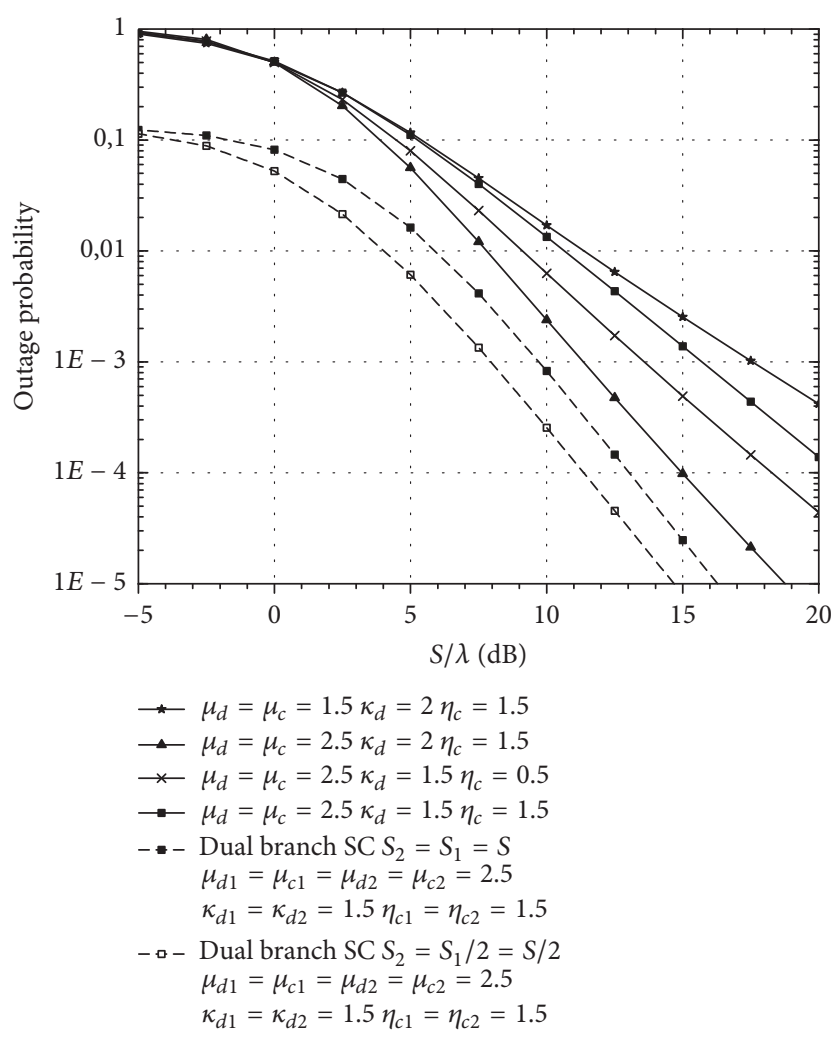

FIGURE 6: Outage probability versus normalized SIR.

fading, so that influences desired and CCI signal are less severe, namely, when parameters $\mu_{d}$ and $\mu_{c}$ take higher values. With SC reception performance improvement reached is also shown. Considerably lower OP values are obtained, comparing to the case with no-diversity reception. Finally it can be seen how balanced reception leads to achieving better quality of service. For example, it can be seen from the figure that when SC diversity has been used, for reaching the same level of OP (i.e., $10^{-4}$ ), it is necessary to apply about $4 \mathrm{~dB}$ lower level power at the transmission, comparing to the level of transmitting power necessary to reach given OP level for the case of single branch reception when same values of propagation parameters are considered. Also, it can be seen from the figure that when the same level of transmitted signal power exists (i.e., $12 \mathrm{~dB}$ ) higher level of outage (i.e., $10^{-3}$ ) is obtained for single branch reception compared to case when SC diversity has been applied (i.e., $10^{-4}$ ), when same values of propagation parameters are considered. Presented efficient numerical methods in such way could enable wireless system designers to perform tradeoff studies among the required values of system performances at the reception (quality of service) in order to determine the optimal choice of transmission parameters in the presence of their available constraints.

\section{Conclusion and Future Work}

In this paper the exact PDF and CDF expressions of the square ratio of $\kappa-\mu$ and $\eta-\mu$ random processes were derived.
Also, a verification of accuracy of these PDF and CDF expressions was given by comparing with the corresponding approximations obtained by the high-precision quadrature formulas of Gaussian type with respect to the weight functions on $(0,+\infty)$. The computational procedure of such quadrature rules is provided by using the contemporary constructive theory of orthogonal polynomials and the corresponding software. Our efficient numerical methods provide necessary control of level of error for the obtained approximation expressions. Also, the convergence of the proposed expressions is secured. Based on the provided analysis of this novel random process, interference-limited wireless communication subjected to the influence of $\kappa-\mu$ fading was observed when undesired occurrence of CCI has been modelled with $\eta-\mu$ random process. Rapid converging infinite-series expressions are presented for received SIR's PDF and CDF. Major communication performance measure, $\mathrm{OP}$, has been calculated in terms of transmission parameters. OP upgrade accomplished by SC reception usage has also been analyzed.

Contribution presented in this paper provides potentials for applying proposed efficient numerical methods in carrying out analysis of other important wireless telecommunication performance measures for observed propagation scenario, such as channel capacity (CC) and average bit error probability (ABER). Closed-form expressions for ABER values could be derived when both coherent and noncoherent modulation techniques are applied for wireless propagation over $\eta-\mu$ interference-limited channels. In a similar manner $\mathrm{CC}$ values could be efficiently evaluated for proposed propagation scenario. By using provided high-precession approximation techniques that are based on presented quadrature formulas of Gaussian type, a verification of accuracy of these ABER and CC expressions could also be provided. Finally, presented approximations could find their application in many fields of telecommunication theory, since proposed solutions can be applied for performance analysis of large number of wireless transmission scenarios.

\section{Conflicts of Interest}

The authors declare that they have no conflicts of interest.

\section{Acknowledgments}

This work is supported in part by the Serbian Academy of Sciences and Arts (Project no. Ф-96) and by the Serbian Ministry of Education, Science and Technological Development (Projects nos. OI 174015 and III 44006).

\section{References}

[1] S. Panić, M. Stefanović, J. Anastasov, and P. Spalević, Fading and Interference Mitigation in Wireless Communications, CRC Press, Taylor \& Francis Group, USA, 2013.

[2] I. S. Gradshteyn and I. M. Ryzhik, Tables of Integrals, Series and Products Academic, New York, 1980.

[3] J. C. S. Santos Filho and M. D. Yacoub, "Highly accurate $\kappa-\mu$ approximation to sum of $\mathrm{M}$ independent non-identical Ricean 
variates," IEEE Electronics Letters, vol. 41, no. 6, pp. 338-339, 2005.

[4] S. R. Panic, D. M. Stefanović, I. M. Petrović, M. Č. Stefanović, J. A. Anastasov, and D. S. Krstić, "Second-order statistics of selection macro-diversity system operating over Gamma shadowed $\kappa-\mu$ fading channels," EURASIP Journal on Wireless Communications and Networking, vol. 2011, article 151, 2011.

[5] P. Stavroulakis, Interference Analysis and Reduction for Wireless Systems, Artech House, Inc., London, 2003.

[6] D. B. Da Costa and M. D. Yacoub, “The $\eta-\mu$ Joint phase-envelope distribution," IEEE Antennas and Wireless Propagation Letters, vol. 6, pp. 195-198, 2007.

[7] P. Spalevic, M. Stefanovic, S. R. Panic, B. Milosevic, and Z. Trajcevski, "The co-channel interference effect on average error rates in Nakagami-q (Hoyt) fading channels," Revue Roumaine des Sciences Techniques. Série Electrotechnique et Énergétique, vol. 56, no. 3, pp. 305-313, 2011.

[8] M. Austin and G. Stuber, "In-service signal quality estimation for TDMA cellular systems," in Proceedings of the 6th International Symposium on Personal, Indoor and Mobile Radio Communications, pp. 836-840, Toronto, Ont., Canada.

[9] W. Gautschi, "On generating orthogonal polynomials," SIAM Journal on Scientific Computing, vol. 3, no. 3, pp. 289-317, 1982.

[10] W. Gautschi, Orthogonal Polynomials, Oxford University Press, 2004.

[11] A. S. Cvetković and G. V. Milovanović, "The Mathematica package OrthogonalPolynomials," Facta Universitatis, Series: Mathematics and Informatics, no. 19, pp. 17-36, 2004.

[12] G. V. Milovanović and A. S. Cvetković, "Special classes of orthogonal polynomials and corresponding quadratures of Gaussian type," Mathematica Balkanica, vol. 26, no. 1-2, pp. 169184, 2012.

[13] K. Peppas, "Dual-hop relaying communications with cochannel interference over $\eta-\mu$ fading channels," IEEE Transactions on Vehicular Technology, vol. 62, no. 8, pp. 4410-4115, 2013.

[14] J. F. Paris, "Outage probability in $\eta-\mu / \eta-\mu$ and $\kappa-\mu / \eta-\mu$ interference-limited scenarios," IEEE Transactions on Communications, vol. 61, no. 1, pp. 335-343, 2013.

[15] A. C. Moraes, D. B. Da Costa, and M. D. Yacoub, "An outage analysis of multibranch diversity receivers with cochannel interference in $\alpha-\mu, \kappa-\mu$, and $\eta-\mu$ Fading scenarios," Wireless Personal Communications, vol. 64, no. 1, pp. 3-19, 2012.

[16] M. Stefanovic, N. Sekulovic, M. Gligorijevic, M. Bandjur, P. Spalevic, and Z. Popovic, "Statistics of signal envelope in composite multipath fading/shadowing microcellular environment," Technics Technologies Education Management, vol. 6, no. 4, pp. 1147-1151, 2011.

[17] G. V. Milovanović, Numerical Analysis, University of Niš, Niš, 1979.

[18] G. V. Milovanović, "Summation of series and Gaussian quadratures," in Approximation and Computation, R. V. M. Zahar, Ed., vol. 119, pp. 459-475, Birkhäuser Boston, Boston, MA, 1994.

[19] G. V. Milovanović, "On summation/integration methods for slowly convergent series," Studia. Universitatis Babeş-Bolyai Mathematica, vol. 61, no. 3, pp. 359-375, 2016.

[20] M.-J. Luo, G. V. Milovanovic, and P. Agarwal, "Some results on the extended beta and extended hypergeometric functions," Applied Mathematics and Computation, vol. 248, pp. 631-651, 2014.

[21] G. V. Milovanović and A. S. Cvetković, "Gaussian-type quadrature rules for Müntz systems," SIAM Journal on Scientific Computing, vol. 27, no. 3, pp. 893-913, 2005.
[22] G. V. Milovanović, "Müntz orthogonal polynomials and their numerical evaluation," in Applications and computation of orthogonal polynomials, W. Gautschi, G. H. Golub, and G. Opfer, Eds., vol. 131, pp. 179-194, Birkhäuser, Basel, 1999.

[23] G. V. Milovanović and A. S. Cvetković, "Nonstandard Gaussian quadrature formulae based on operator values," Advances in Computational Mathematics, vol. 32, no. 4, pp. 431-486, 2010.

[24] G. V. Milovanović and A. S. Cvetković, "Gaussian quadrature rules using function derivatives," IMA Journal of Numerical Analysis (IMAJNA), vol. 31, no. 1, pp. 358-377, 2011.

[25] G. V. Milovanović, "Weighted quadrature formulas for semiinfinite range integrals," Journal of Numerical Analysis and Approximation Theory, vol. 44, no. 1, pp. 69-80, 2015.

[26] G. Mastroianni and G. V. Milovanović, Interpolation Processes: Basic Theory and Applications, Springer, 2008.

[27] G. H. Golub and J. H. Welsch, "Calculation of Gauss quadrature rules," Mathematics of Computation, vol. 23, no. 106, article 221, 1969.

[28] G. V. Milovanović and A. S. Cvetković, "Note on a construction of weights in Gauss-type quadrature rule," Facta Universitatis, Series: Mathematics and Informatics, no. 15, pp. 69-83, 2000.

[29] G. V. Milovanović, M. M. Spalević, and A. S. Cvetković, "Calculation of Gaussian-type quadratures with multiple nodes," Mathematical and Computer Modelling, vol. 39, no. 2-3, pp. 325347, 2004.

[30] R. P. Agarwal and G. V. Milovanović, "Extremal problems, inequalities, and classical orthogonal polynomials," Applied Mathematics and Computation, vol. 128, no. 2-3, pp. 151-166, 2002.

[31] G. B. Djordjević and G. V. Milovanović, Special Classes of Polynomials, University of Niš, Faculty of Technology, Leskovac.

[32] G. V. Milovanović, "Construction and applications of Gaussian quadratures with nonclassical and exotic weight functions," Studia. Universitatis Babeş-Bolyai Mathematica, vol. 60, no. 2, pp. 211-233, 2015.

[33] G. V. Milovanović, "Symbolic-numeric computation of orthogonal polynomials and Gaussian quadratures with respect to the cardinal B-spline," Numerical Algorithms, vol. 76, no. 2, pp. 333347, 2017.

[34] M. Masjed-Jamei and G. V. Milovanović, "Construction of Gaussian quadrature formulas for even weight functions," Applicable Analysis and Discrete Mathematics, vol. 11, no. 1, pp. 177-198, 2017.

[35] W. Gautschi, Orthogonal Polynomials in Matlab: Exercises and Solutions, Software - Environments - Tools, SIAM, Philadelphia, PA, USA, 2016.

[36] M. Č. Stefanović, D. M. Milović, A. M. Mitić, and M. M. Jakovljević, "Performance analysis of system with selection combining over correlated Weibull fading channels in the presence of cochannel interference," AEÜ - International Journal of Electronics and Communications, vol. 62, no. 9, pp. 695-700, 2008.

[37] D. Milic, S. Stanojcic, Z. Popovic, D. Stefanovic, and I. Petrovic, "Performance analysis of EGC combining over correlated Nakagami-m fading channels," Serbian Journal of Electrical Engineering, vol. 9, no. 2, pp. 293-300, 2012.

[38] M. K. Simon and M. S. Alouini, Digital Communication over Fading Channels, John Wiley \& Sons, USA, 2000. 


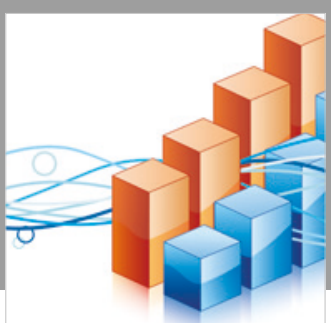

Advances in

Operations Research

\section{-n-m}
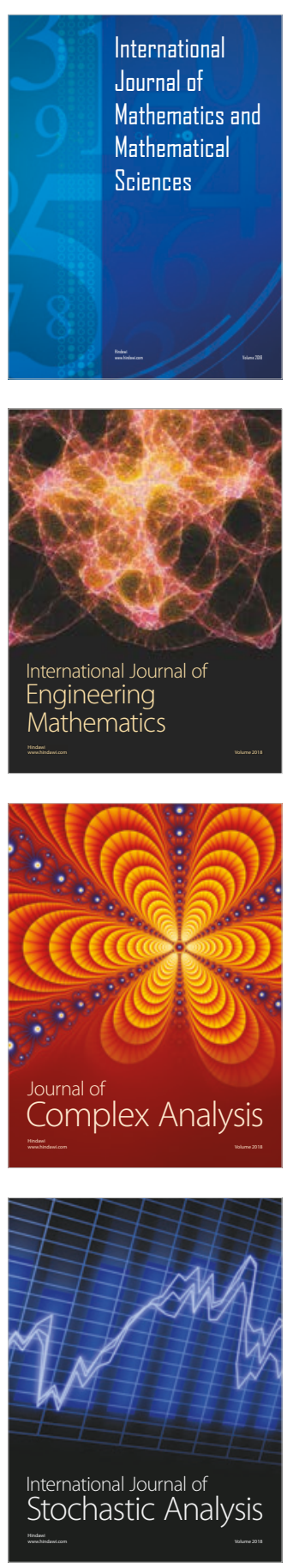
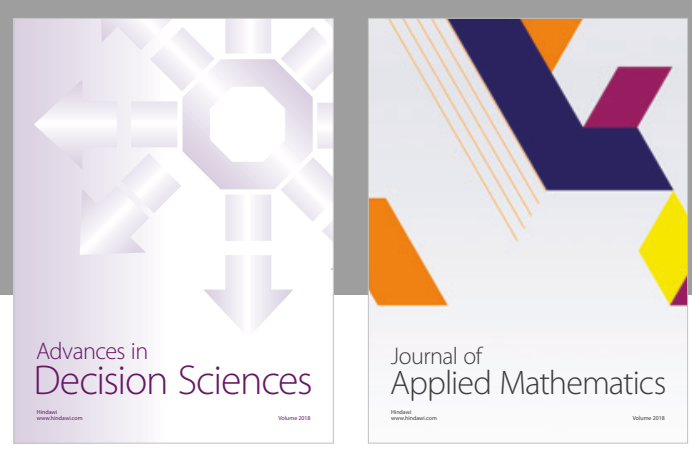

Journal of

Applied Mathematics
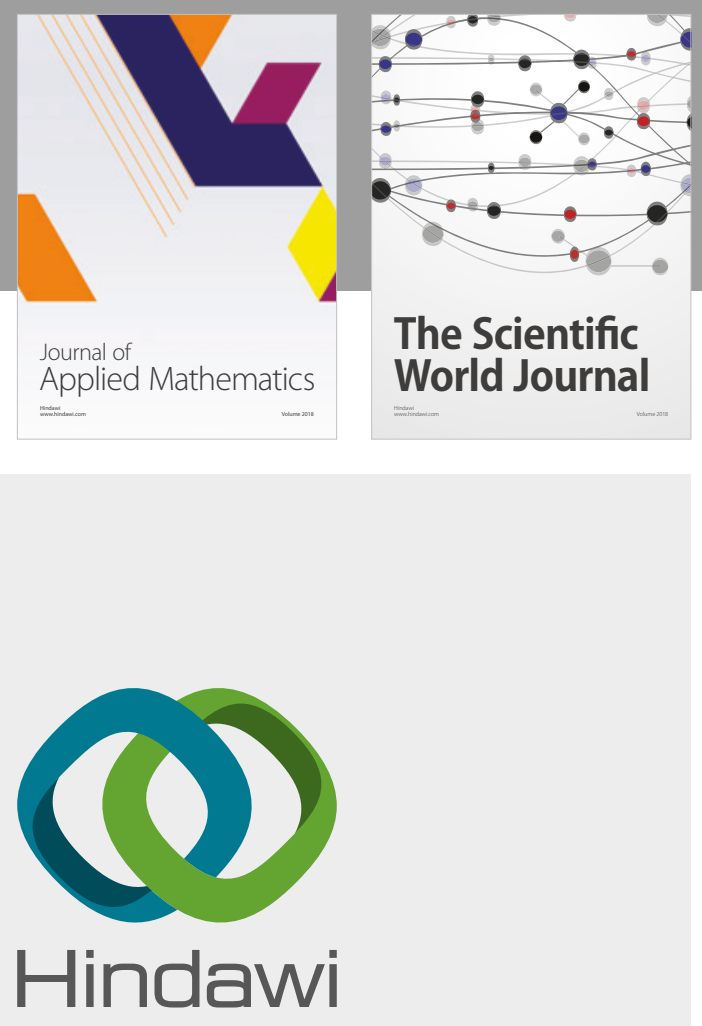

Submit your manuscripts at

www.hindawi.com

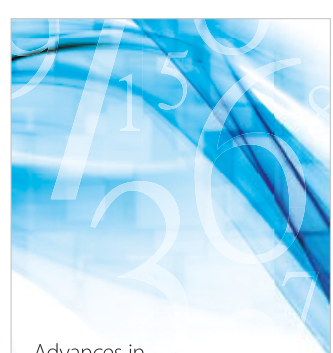

Advances in
Numerical Analysis
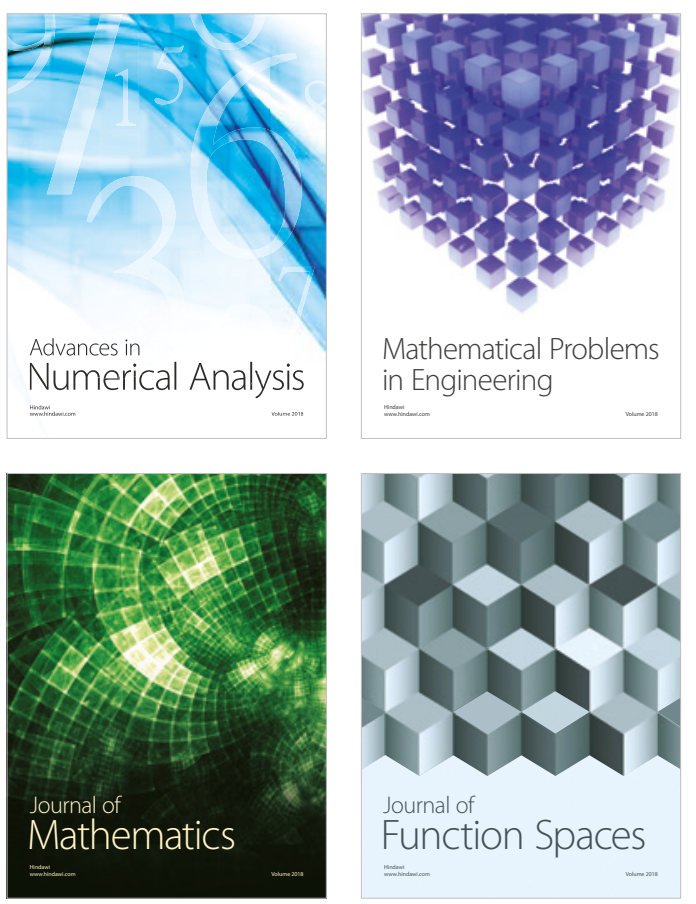

Mathematical Problems in Engineering

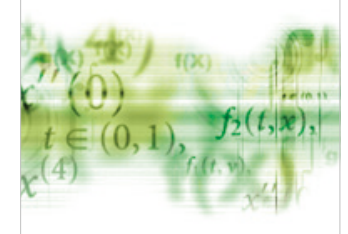

International Journal of

Differential Equations

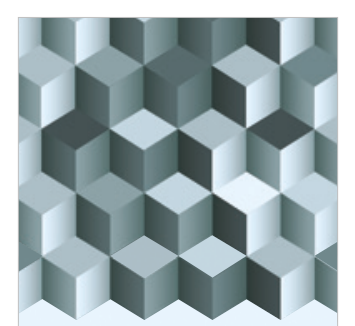

Journal of

Function Spaces

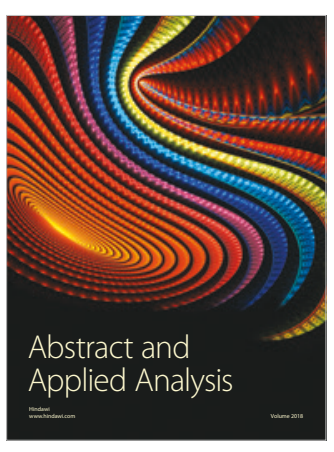

The Scientific

World Journal

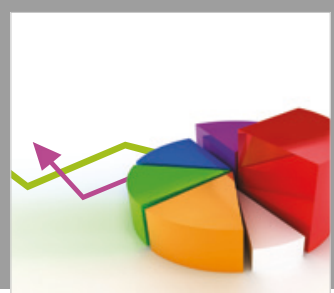

Journal of

Probability and Statistics
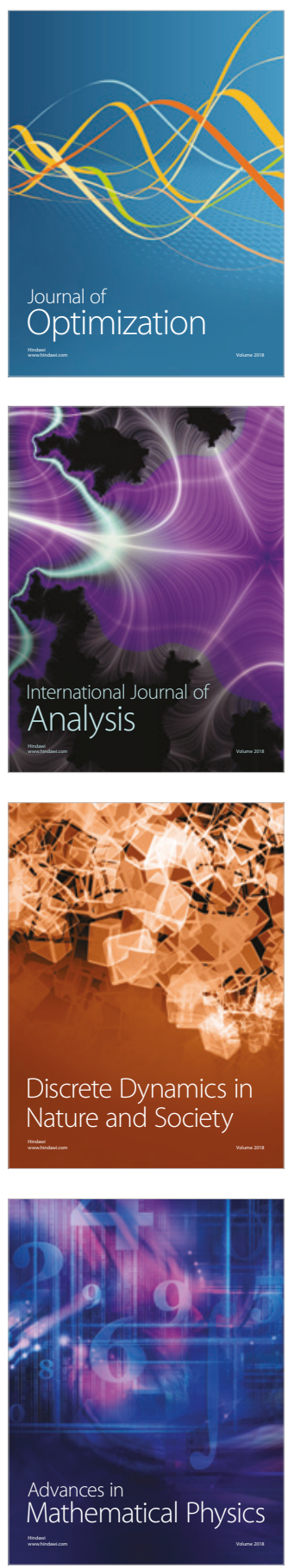